\title{
LA REHABILITACIÓN DE LA NOCIÓN DE REPRESENTACIÓN EN TOULMIN (O UN INTENTO DE REFORMULAR A KANT EN TÉRMINOS WITTGENSTEINIANOS)
}

\section{María Inés Prono*}

\section{Los avatares de la noción de representación}

La noción de representación aplicada a los procesos cognoscitivos, ha sufrido diversos ataques que pretenden desacreditarla como categoría útil en la comprensión de los fenómenos gnoseológicos. Las llamadas corrientes 'antirrepresentacionalistas', con Rorty como uno de sus exponentes paradigmáticos, han sostenido la obsolencencia de este concepto, por hallarse atado a lo que consideran la herencia más retrógrada de la filosofía occidental ${ }^{1}$. La serie de dicotomías listadas habitualmente por Rorty, a saber: espacio interior vs. espacio exterior; real vs. aparente; encontrado vs. hecho; absoluto vs. relativo, que son usadas para ejemplificar estos obstáculos, le sirven de guía para sostener que el abandono de las mismas permite "hacer a un lado tanto a Platón como a Kant" y encontrar "el lazo que vincula la tradición posnitzscheana de la filosofía europea con la tradición pragmática norteamericana"2.

Por este camino, sostiene, será posible reemplazar o sustituir dichas dicotomías por la distinción entre lo más útil y lo menos útil, y seguir a Wittgenstein en el intento de romper con la "imagen que nos mantiene cautivos", la imagen cartesiano-lockeana de una mente que procura ponerse en contacto con una realidad exterior a ella. Por el contrario, las palabras tanto como las creencias cuentan como herramientas. "Ver el empleo de palabras como el uso de herramientas para manejarse con el medio, y no

\footnotetext{
*Profesora de la Universidad Nacional del Litoral (miprono@ciudad.com.ar).

${ }^{1}$ Es clásica ya la posición antrrepresentacionalista que Rorty presenta en I a filosofía y el espejo de la naturaleza (1979) Madrid, Cátedra, 1989.

${ }^{2}$ Rorty, R. (1998) «Relativismo: el encontrar y el hacen» en Giro linguístico e bistoria intelectual. Elías J. Palti (ed.) Buenos Aires, U.N.Q., 1998, p. 296 (En adelante REH).
} 
como un intento de representar la naturaleza intrínseca de ese medio, significa rechazar la cuestión de si las mentes humanas están en contacto con la realidad, la cuestión que plantea el escéptico epistemológico. ... Abordar la creencias no como representaciones sino como hábitos de acción y las palabras no como representaciones sino como herramientas, es hacer que resulte inútil preguntar "¿descubriendo o inventando, haciendo o encontrando?"3.

De este modo, la utilidad a nuestros propósitos y la relatividad de nuestras descripciones son, para Rorty, "el principal argumento del pragmatista a favor de su visión antirrepresentacional del conocimiento -la visión de que la investigación apunta a la utilidad para nosotros más que a una descripción precisa de cómo son las cosas en sí mismas"4.

No es difícil ver que Rorty se maneja con una noción empobrecida o sesgada de las representaciones, que las asocia inmediatamente con un fenómeno mental y privado, que perseguiría el objetivo principal de describir la realidad tal cual es en sí misma. En esta caracterización, la noción de representación aparece indisociablemente unida a una concepción realista epistemológica en sentido fuerte, según la cual el conocimiento sería un intento por descubrir la verdad acerca de lo que hay en un mundo real único, independientemente del pensamiento o las operaciones de las personas que llevan a cabo el conocimiento.

En lo que sigue, no es nuestra intención polemizar con Rorty sino tratar de mostrar que es posible mantener el valor de la noción de representación para el análisis gnoseológico y epistemológico. Al mismo tiempo, la defensa de la utilidad de la noción de representación no implica necesariamente la adopción de tesis realistas en sentido fuerte, que se han vuelto enormemente problemáticas en los últimos tiempos, sino que admite un tratamiento pragmático de las mismas y de la manera en que se constituye su significado, de modo que también supone alejarse del tratamiento de las representaciones vinculadas a la concepción semántico-referencialista, propias del realismo semántico.

\footnotetext{
${ }^{3}$ Cfr. REH, p.303-307.

${ }^{4}$ REH, p. 308.
} 
Para este fin, analizaremos los aportes de Toulmin realizados en La comprensión bumana. El uso colectivo y la evolución de los conceptos, en la que considera que la noción de representación no sólo es útil sino imprescindible en relación al objetivo de comprender la naturaleza de la actividad científica o cognoscitiva en general ${ }^{5}$. Comparte con Kant la idea de que nuestras actividades intelectuales imponen formas a priori a nuestros juicios $\mathrm{y}$, por ende, a nuestro conocimiento. Pero se preocupa por hacer hincapié en el carácter público e histórico de nuestras representaciones, mostrando que las mismas constituyen un elemento fundamental en la mejora de nuestros modos de explicar, comprender e interpretar la realidad. También se permite reinterpretar a Kant en términos de procedimientos, dándole así un "giro wittgensteiniano".

\section{Los conceptos en el planteo de Toulmin}

Toulmin se propone explicar el desarrollo y evolución históricos de las disciplinas científicas. Para este fin plantea acercarse a las disciplinas concibiéndolas como poblaciones conceptuales antes que como sistemas estructurados a partir de relaciones lógicas rígidas. En este contexto la noción de concepto no sólo aparece como categoría fundamental, sino que es caracterizada de un modo peculiar.

La primera aclaración que hace Toulmin es que el análisis filosófico de los conceptos tendrá que ver fundamentalmente, no con los procesos de adquisición que involucran a nivel individual (lo cual constituiría el objetivo de una explicación psicológica de los mismos), sino con los aspectos colectivos del uso de los conceptos en las disciplinas intelectuales y en la vida social en general. Esto implica que no se tratará a los conceptos como

${ }^{5}$ Lamentablemente, sus propuestas han sido prácticamente ignoradas en la literatura epistemológica de los últimos treinta años. Creemos que las razones de este olvido se deben, en parte, al fuerte impacto que produjo la obra de Kuhn en la epistemología, que eclipsó la producción teórica a partir de los problemas que abría en relación a la explicación del cambio científico; por otro lado, la concepción evolutiva que allí presentó tampoco se encuentra exenta de dificultades en relación al objetivo anterior, y por último, él mismo no ha continuado esta línea de indagaciones, a tal punto que nunca escribió la segunda parte de la mencionada obra, de la que hace explícitas promesas en el prólogo. 
representaciones 'internas', sino como representaciones 'externas', o públicas, que son usadas por los miembros de una comunidad epistémica para llevar adelante sus procesos explicativos, y destaca que los aspectos públicos del uso de los conceptos son determinantes de su sentido.

Se parte de la idea de que las actividades desarrolladas por quienes conocen, suponen modos de estructurar la experiencia, formas de categorizar los objetos que abordamos y representaciones simbólicas (principalmente el lenguaje) para nombrar, generalizar y establecer relaciones entre los fenómenos. Todas estas funciones son llevadas a cabo por los conceptos, aunque estos no se identifican con ninguna de ellas en particular. Por esta razón, los conceptos son entidades complejas, compuestas por elementos de diferente índole, todos los cuales son necesarios para una adecuada comprensión de los mismos, pero que, a la vez, mantienen entre sí un cierto grado de independencia relativa.

"Para hacer justicia a la complejidad de los conceptos científicos debemos distinguir tres aspectos o elementos en el uso de tales conceptos: I) el lenguaje; II) las técnicas de representación, y III) los procedimientos de aplicación de la ciencia. Los primeros dos elementos comprenden los aspectos «simbólicos» de la explicación científica ..., mientras el tercero comprende el reconocimiento de las situaciones a las que son apropiadas esas actividades simbólicas"6.

Según lo anterior, los conceptos no se reducen a los términos ni a las oraciones que expresan leyes o generalizaciones. Estos representan el aspecto lingüístico del concepto, que forma parte de la faz simbólica. El segundo de los elementos, son las técnicas de representación. Con esta expresión designa a todos aquellos procedimientos que los científicos utilizan para acompañar o apoyar sus explicaciones: formalismos matemáticos, gráficos y diagramas, árboles taxonómicos, programas de computación, y podríamos agregar los modelos de simulación, etc. El tercero, los procedimientos de aplicación, no supone sólo una aptitud lingüística, o semántica, sino también criterios y aptitudes para someter a prueba y delinear el "ámbito" o "rango de

${ }^{6}$ Toulmin, S. (1972) La comprensión bumana. Volumen I: El uso colectivo y la evolución de los conceptos, Madrid, Alianza, 1977, p. 170, (resaltado en el original). En adelante se citará $\mathrm{CH}$, seguido del número de página. 
aplicación" dentro de los cuales esos símbolos y técnicas de representación tienen relevancia empírica. Vemos así que los conceptos imponen una especie de relación dialéctica que conecta aspectos lingüísticos y empíricos de las disciplinas. "En la solución de problemas conceptuales, los elementos semánticos y los empíricos no se hallan tanto arbitrariamente confundidos como inevitablemente fundidos" ". Así, por ejemplo, la aparición de un nuevo término técnico tiene que ver con el cambio en las actividades de explicación, ya que es necesario destacar, las técnicas de representación son las que hacen posible determinar el ámbito de aplicación de esos conceptos.

\section{Sobre el carácter de la noción de representación}

Como exponente de la orientación post-empirista, Toulmin quiere alejarse de las posiciones gnoseológicas o epistemológicas tradicionales, dejando de la lado las metáforas espaciales interno-externo heredadas del racionalismo y el empirismo modernos que en gran medida se conservaron en la epistemología de la primera mitad del S. XX. Sin embargo, podríamos decir que se inclina claramente por uno de los aspectos de la dicotomía: el externo, ya que lo que interesa explicar son los aspectos transmisibles de disciplinas colectivas, que se reflejan a través de sus procedimientos explicativos.

Comenta que el término 'representación' -que fue puesto en circulación por Kant y luego por Schopenhauer- emparenta dos nociones conectadas entre sí, que no siempre se han distinguido claramente. Durante el siglo XIX, en la óptica fisiológica de Helmholtz y en la psicología de Mach, el término tenía un empleo «sensorial» o "perceptual», que lo vinculaba con las filosofías empiristas de Locke y Hume. En su otro sentido, tenía un empleo más "público» o «lingüístico» -mecánica de Hertz- análogo al de 'representación gráfica' de la física actual. En general el empleo «sensorial» estaba emparentado con el término alemán Vorstellung, el empleo «público», con la palabra Darstellung8.

La noción de representación en Kant parece estar usada para significar una facultad o capacidad; una actividad que establece relaciones y que indica

\footnotetext{
${ }^{7} \mathrm{CH}$, p.197.

${ }^{8}$ Cfr. CH, p. $199-205$.
} 
que es precisa la espontaneidad para recibir datos. En ciertos contextos, el uso del término representación por parte de Kant, puede haber estimulado la interpretación decimonónica de las mismas como "representaciones sensoriales", o al menos como algún tipo de entidad psicológica perteneciente al 'espacio interior'. Por ejemplo en la Estética Trascendental:

"Las representaciones en las que no se encuentra nada perteneciente a la sensación las llamo puras (en sentido trascendental). Según esto, la forma pura de las intuiciones sensibles en general, donde se intuye en ciertas relaciones toda la diversidad de los fenómenos se hallará a priori en el psiquismo" (A 20, B 34) .

Sin embargo, la aclaración de que se trata de un sentido trascendental, debe hacernos cautos. Significa que se trata de una condición universal y necesaria que posibilita la experiencia. En otros pasajes el uso de 'representación' cumple claramente con otra finalidad. Kant puso énfasis en el papel que desempeñan las «formas del juicio» en la tarea de dar una «estructura» al conocimiento. Las formas lógicas (o lingüísticas) del juicio son asimismo las formas de toda «experiencia» genuina. Nuestras propias experiencias sensoriales se nos presentan con una estructura epistémica reglada; a esta estructura sólo se la puede caracterizar en términos de formas del juicio ${ }^{10}$.

"Como ninguna representación que no sea intuición se refiere inmediatamente al objeto, jamás puede un concepto referirse inmediatamente a un objeto, sino a alguna otra representación de éste último (...). El juicio es, pues, el conocimiento mediato de un objeto, por consiguiente, representación de una representación del objeto" (A 68, B 93).

${ }^{9}$ Kant, I. Crítica de la razón pura. Trad. Pedro Ribas. Madrid, Alfaguara, 2003. (Se citará según esta traducción, indicando la referencia a A y/o B).

${ }^{10}$ Coincidimos con la observación de Ibarra e Uribarri: "El interés de Kant por la representación es primordialmente no tanto de cómo esta se produce cuanto del papel que representa en la justificación del conocimiento. Su meta es explicar el carácter objetivo del conocimiento ... su interés se centra en la vertiente activa de la representación: es precisa la espontaneidad para recibir datos (Opus postumun, XXI, 52, 1-3)". Andoni Ibarra e Ibon Uribarri, «El reto del representacionalismo empirista en Kant» en Variedades de la representación en la ciencia y la filosofia, A. Ibarra y Th. Mormann (eds.). Barcelona, Ariel, 2000, p. 62. 
"Según esto, todos los conceptos son funciones de unidad entre nuestras representaciones. En efecto, para conocer el objeto se utiliza, en vez de una representación inmediata, otra superior, la cual comprende en sí la anterior y otras más; de esta forma se sintetizan muchos conocimientos posibles en uno solo" (A 69, B 94).

Retomando este aspecto es que Toulmin propone que conservemos de Kant su recurso a las representaciones, ya que esto ha permitido evitar la esterilidad de la discusión epistemológica tradicional, pero que reformulemos a Kant en términos de «procedimientos». Por esta vía se evita la ambigüedad del término Vorstellung y se puede mostrar que el carácter de nuestras actividades intelectuales impone formas a priori a nuestros juicios y, por ello, a nuestro conocimiento.

"Su objetivo [el de Kant] era, en parte, destacar la estructura formal o gramatical de todos los juicios y la dependencia de todo conocimiento respecto de los conceptos y las formas intelectuales particulares en términos de los cuales se expresan los juicios resultantes. Desde el punto de vista de Kant, todos los juicios sobre la naturaleza de los objetos externos y todo conocimiento acerca de su conducta deben ser especificados en forma proposicional; lo que sabemos o lo que juzgamos es siempre "que sucede tal y tal cosa"11.

Es visible que Toulmin hace aquí una interpretación de Kant tamizada por la mediación del giro lingüístico y por su lectura de Wittgenstein. Siguiendo con su tratamiento de las expresiones alemanas, sugiere que si sustituimos 'representación' por Darstellung y 'representar' por darstellen, que tienen el sentido de una representación pública, la relación entre una Darstellung y la realidad que «despliega» o «representa» es, por consiguiente, una relación entre dos entidades públicas. Difícilmente esto pueda ser aplicado a Kant. Aún cuando para él las formas del juicio eran objetivas, lejos está esto de significar 'públicas'. Por otra parte, el tratamiento proposicional del conocimiento, si bien podría derivarse del planteo kantiano, es un paso que efectivamente Kant no dio, quizás porque no estaban dadas las condiciones intelectuales para que lo diera.

${ }^{11} \mathrm{CH}$, p. 200. 
"En Wittgenstein el giro reflexivo, que está solamente implícito en Kant, toma un estado central, y es radicalmente transformado. En parte inspirado por Schopenhauer y Hertz, transforma el problema kantiano acerca de cómo experimentamos los objetos en el problema de cómo representamos o describimos la realidad. De acuerdo a Kant, representamos la realidad teniendo 'representaciones' (Vorstellungen). En Wittgenstein, por contraste, 'representación' (Darstellung, Abbildung) tiene connotaciones lógicolingüísticas antes que mentalistas: representamos la realidad por la construcción de modelos (Bilder. TLP 2.1)"12.

\section{E1 "giro wittgensteiniano"}

En su lectura del Tractatus de Wittgenstein ${ }^{13}$, Toulmin objeta la traducción del término Bild y sus formas verbales asociadas abbilden, por 'pintura', ya que esta sugiere considerar que las «proposiciones» nos proporcionan algo así como fotografías instantáneas -o incluso imágenes mentales- de los «hechos». Las observaciones de Wittgenstein sobre la relación que hay entre proposiciones y hechos, están expresadas en términos activos, constructivos. Un Bild o «representación» es para Wittgenstein, algo que nosotros hacemos o producimos, como un artefacto. Cuando Wittgenstein declaraba que las proposiciones del lenguaje representan (darstellen) los hechos, sus afirmaciones no aludían a nada específicamente «mentab» o «interno». ${ }^{14} \mathrm{Al}$ equiparar las oraciones con figuras pretendía destacar que la figura o Bild es un hecho físico, y las oraciones como figuras eran bechos físicos; pues se consideró una apreciación lógica importante el que la figura fuese no una cosa sino un hecho.

En la siguiente selección y relación de aforismos del Tractatus, lo presentamos de un modo esquemático:

${ }^{12}$ Glock, Hans-Johan. «Kant and Wittgenstein: Philosophy, Necesity and Representation». International Journal of Philosopbical Studies Vol. 5 (2), 285-305. Routledge, 1997, p. 291.

${ }^{13}$ Wittgenstein, L. Tractatus Logico-Philosophicus. (TLP) Ed. bilingüe. Trad. de Jacobo Muñoz e Isidoro Reguera. Madrid, Alianza, 1987. Las citas se realizan de acuerdo a esta edición, indicando solamente la numeración de los parágrafos. ${ }^{14}$ Cfr. TLP, 4.1 "La proposición representa el darse y no darse efectivo de estados de cosas". 
"2.1 Nos hacemos figuras de los hechos."

"2.12 La figura es un modelo de la realidad."

"2.19 La figura lógica puede figurar el mundo."

Toulmin apoya su argumento agregando que existe una importante continuidad entre el término Bild tal como lo usó Hertz en la mecánica, como «modelo», y el uso wittgensteniano; y sostiene que el orden espacial que aparece en una pintura es muy distinto del orden lógico que caracteriza a los modelos teóricos o matemáticos de la ciencia natural. Desde este punto de vista, los Bilder son «representaciones», pero no representaciones psicológicas, subjetivas o internas (Vorstellungen). Los modelos de Wittgenstein son representaciones en el sentido de Darstellungen, y pretende resaltar el carácter de construcción de los modelos, y su diferencia con las reproducciones que proceden de la experiencia sensorial, ya que un modelo:

"2.1512 Es como un patrón de medida aplicado a la realidad".

Es posible interpretar, entonces, que los modelos podrían ser un sistema a priori capaz de modelar el mundo entero, de proveer la estructura lógica de toda descripción, en virtud de la estructura isomórfica entre modelo y realidad. ¿De qué manera modelan los modelos (Bilder) lingüísticos? Las proposiciones son representaciones que hacemos de los hechos, pero no son representaciones exactas de ellos, sino sólo de lo que es esencial en ellos.

Haciendo este giro wittgensteniano, Toulmin sostiene que aún así se conserva "buena parte de la tesis central de Kant, según la cual el conocimiento es incapaz de captar las «cosas en sí»; en la medida en que el contenido del conocimiento sólo puede ser especificado en las formas del juicio, lo que se conoce no es un objeto independiente del pensamiento humano, sino un hecho o una proposición lingüísticamente estructurados"15.

Toulmin tampoco puede ser considerado un realista epistemológico, ya que sostiene que la conclusión anterior estaba presente en la observación lógica de Kant de que "todo nuestro conocimiento lo es de representaciones." 16 Sin embargo, esto no significa negar la independencia de

${ }^{15} \mathrm{CH}$, p. 203.

${ }^{16} \mathrm{CH}, \mathrm{P} .181$ 
la realidad (conocida) de una mente individual o de las actividades cognitivas individuales, ya que esto haría imposible explicar la naturaleza intersubjetiva de las actividades cognoscitivas. Las cosas existen y actúan independientemente de nuestras descripciones, pero sólo podemos conocerlas bajo ciertas descripciones.

En referencia a la cuestión acerca del significado de los conceptos, no es preciso insistir en el carácter anti-psicologista que Toulmin quiere otorgarle a los conceptos y a las representaciones como elementos fundamentales de los mismos y de su significado. Se señaló antes la estrecha conexión existente entre los aspectos lingüísticos y empíricos de las disciplinas y el modo cómo se funden los elementos semánticos y empíricos en la determinación del ámbito de aplicación de los conceptos.

En este sentido, aunque Toulmin no tematizó la cuestión en esa obra, podemos decir que no es un realista semántico, que sostenga que hay una relación referencial objetiva entre los términos y enunciados lingüísticos y las entidades no lingüísticas. El núcleo del realismo semántico sería la idea de que las oraciones de una teoría son verdaderas o falsas en virtud de "cómo es el mundo independientemente de nosotros", o dicho de otro modo, la interpretación realista del significado se presenta en términos de condiciones de verdad: conocer el significado de un enunciado concreto es saber qué tendría que ocurrir en el mundo para que el enunciado fuera verdadero. Al respecto, Toulmin sostiene que: "... los filósofos han supuesto que los términos usados en las teorías científicas se refieren directamente a clases de objetos ... . Nuestro enfoque, en cambio, implica que este supuesto básico es totalmente incorrecto, ya que en la ciencia no se plantean cuestiones concernientes a la verdad o falsedad empírica de los principios teóricos. En cambio, los términos y enunciados teóricos adquieren un contenido o referencia empírico indirecto sólo cuando se ha hecho explícito su ámbito o dominio de aplicación mediante enunciados de identificación auxiliares ..." 17

Visto de este modo, el problema del significado de los conceptos científicos, será un problema que excede ampliamente el ámbito de los términos, oraciones o teorías. Involucra al resto de las actividades disciplinarias con fines explicativos, y se convierte en un problema conceptual

${ }^{17} \mathrm{CH}$, p. 178 (resaltado en el original). 
$y$, por ende, en un asunto comunal o público, sujeto a transformación histórica. De modo que los conceptos "adquieren significado sirviendo a fines humanos relevantes en los casos prácticos reales". ${ }^{18}$ Las "técnicas de representación" entendidas como darstellung, exhiben las relaciones entre los fenómenos, las muestran; y el problema del significado es el problema del uso público de los conceptos que suponen esas técnicas de representación; supone también el manejo de toda una red de significados interconectados y la competencia en la utilización de las técnicas de representación. La comprensión del significado por parte de un individuo significará captar y saber usar un repertorio de técnicas, procedimientos, habilidades intelectuales y métodos de representación; y todos estos procesos se encuentran reglados en las disciplinas. La idea es que los conceptos adquieren su significación del uso que hacen los científicos en sus actividades explicativas; y ello indica que su significado es constituido por esas actividades de explicación. Es notorio que la concepción de significado supuesta aquí se acerca a los planteos holísticos y pragmáticos, y que nuevamente nos encontramos cercanos a la concepción de significado como uso del segundo Wittgenstein.

\section{Algunas consideraciones finales}

La visión de la actividad cognitiva que ha sido presentada, nos permite concebirla como una actividad primordialmente representacional, es decir, como una práctica representacional y cognitiva. Tal análisis, no sólo es válido para el contexto de las actividades científicas, sino que es perfectamente extensible al resto de las actividades cognoscitivas en general. Nuestra manera habitual de comprender el mundo y de actuar en él, aunque esté estructurada de un modo diverso al de las ciencias, también es conceptual y está sujeta a variación histórica. Por estas razones es igualmente comunicable de una generación a otra, y aunque moldea e impone improntas a nuestro modo de vivir, de conocer y de decir, es susceptible de ser modificada o recreada por cada generación de usuarios, a raíz de las formas particulares de apropiación y educación.

\section{Ibidem.}


La noción de concepto que maneja Toulmin y, junto con ella, la noción de representación mediatizada por el giro pragmático wittgensteniano, son lo suficientemente complejas y ricas como para no quedar reducidas ni a mera expresión lingüística, ni a un contenido mental individual, cuya existencia y utilidad son dudosamente constatables. Definidas negativamente, las representaciones conceptuales toulminianas no son: (i) entidades que están "detrás" de las expresiones lingüísticas; ni (ii) episodios mentales (à la Descartes o el empirismo clásico); y tampoco (iii) entidades abstractas (como en Platón, Bolzano o Frege). Antes bien, son procedimientos-en-uso, públicos e intersubjetivos, que se proyectan sobre la realidad y que se transforman de acuerdo a las necesidades cognoscitivas de los usuarios respectivos.

Como se ha dejado ver más arriba, la adopción de esta estrategia representacional no implica compromiso alguno con la pretensión realista ontológica de «describir la realidad tal cual es en sí misma», ni con la tesis del realismo semántico de la referencia directa. Las consideraciones pragmáticas acerca de las relaciones entre conocimiento, lenguaje y prácticas humanas ofrecen una alternativa superadora en relación a posiciones reductivas. Fundamentalmente, nos permiten superar las concepciones intelectualistas, racionalistas y objetivistas del conocimiento y el lenguaje humanos. También nos conducen a concebir la realidad como nuestra realidad, es decir, modelada por nuestros modos de conocer, decir y actuar. No ponemos en duda que las cosas existen y actúan independientemente de nuestras descripciones, pero afirmamos que sólo podemos conocerlas bajo ciertas descripciones.

Finalmente, consideramos que la cuestión general acerca de la posibilidad de acceder al conocimiento de objetos que son independientes de cualquier práctica, pensamiento y acción es, prácticamente, una posición epistemológica insostenible. Si se supone que los objetos son independientes de la totalidad de nuestras prácticas, pensamiento y conocimiento, es decir de nuestras representaciones, se sigue que no se podría pensar en ellos. Si hubiera tales objetos, sólo podríamos hacer «silencio» acerca de los mismos. Y no podrían empezar a existir cognitivamente para nosotros, en ningún sentido. 


\title{
Resumen
}

Frente a los ataques de las corrientes "antirrepresentacionalistas", se presenta el análisis de Toulmin, que considera que la noción de representación es imprescindible para comprender la naturaleza de la actividad cognoscitiva. Comparte con Kant la idea de que nuestras actividades intelectuales imponen formas a priori, pero señala que la utilización del término alemán Vorstellung en la obra de Kant, hizo que su objetivo trascendental quedara atrapado en las redes de representaciones internas o mentales.

La noción de representación que maneja, mediatizada por el giro pragmático wittgensteniano, es lo suficientemente compleja como para no quedar reducida a mera expresión lingüística, ni a un contenido mental individual. La adopción de esta estrategia representacional no implica compromiso alguno con la pretensión realista ontológica de «describir la realidad tal cual es en sí misma», ni con la tesis del realismo semántico de la referencia directa. Las consideraciones pragmáticas acerca de las relaciones entre conocimiento, lenguaje y prácticas humanas ofrecen una alternativa superadora en relación a posiciones reductivas.

Palabras clave: “Toulmin", "Kant”, "Wittgenstein", "Representación”

\begin{abstract}
As opposed to the anti-representationalist positions, this work presents Toulmin's analysis. The author considers that the notion of representation is indispensable to understand the nature of cognitive activity. He shares with Kant the idea that our intellectual activities impose a priori forms, but he points out that the use of the German word Vorstellung in Kant's work caused his transcendental goal to get trapped in the webs of mental representations.

The notion of representation he handles, mediated by the wittgensteinian pragmatic turn, is too complex to be reduced to a mere linguistic expression, or to any individual mental content. The adoption of this representational strategy does not entail any commitment to the ontological realist claim "to describe reality as it is in itself", neither to the semantic realist thesis of direct reference. Pragmatic considerations about the relations between knowledge, language and action provide an alternative that overcomes reductive positions.
\end{abstract}

Key words: “Toulmin", “Kant”, “Wittgenstein”, "representation” 